\title{
Strategies for Fast Scanning and Handovers in WiMax/802.16
}

\author{
Paul Boone, Michel Barbeau and Evangelos Kranakis \\ School of Computer Science, Carleton University \\ Ottawa, Canada \\ \{pboone, barbeau, kranakis\}@scs.carleton.ca
}

\begin{abstract}
In WiMax/IEEE 802.16 with mobility support, scanning for an available channel by a mobile station, at start up or when about to perform a handover must be done promptly. We propose strategies that a mobile station can use to reduce the time required for scanning operations while attempting to establish network connectivity or perform a handover between neighboring base stations. We model and simulate an area of WiMax coverage using real-world mobility trace data and show that there are strategies that reduce the time required for scanning operations significantly.
\end{abstract}

\section{INTRODUCTION}

With the neverending growth of mobile communication systems today, mobile devices are becoming a central part of peoples lives. Applications such as VoIP, music downloads and streaming video, are being deployed that utilize an ever increasing chunk of the precious bandwidth available with current $3 \mathrm{G}$ systems. The next generation of $4 \mathrm{G}$ networks are under development and the IEEE 802.16e/WiMax [3] standards are promising to provide the infrastructure for the future of the high-speed mobile internet.

When a mobile subscriber station (MS) wishes to join the network, it must follow the network entry procedure. This involves scanning for a frequency on a base station (BS). It is expected that the MS will be required to perform repeated scanning to maintain connectivity to the network by moving from one BS to another while moving throughout the coverage area. This process of changing BSs is called a handover. The impact of handovers between base stations is a serious problem in a mobile communication system that must be addressed. During a handover, packets may be delayed and connections may be dropped. Real-time applications such as VoIP and streaming video can be adversely affected by these delays.

We address two aspects of a MSs scanning, that of initially finding an available downlink frequency to a base station when entering a network, and that of the scanning a MS must perform when selecting a target BS when it is about to perform a handover.

\section{A. Results of the Paper}

We present several strategies that a MS may use in order to improve scanning times while searching for a downlink from a BS. The strategies attempt to provide faster network access during the network entry phase as well as reduce delay during the handover procedure. The first set of strategies incorporates the history of successful scanning frequencies in order to guide the MS in choosing frequencies for future scanning operations. A second type of strategy improves upon the use of the MOB_NBR-ADV messages sent to the MS from the serving BS that informs the MS of neighbouring BSs. A MS builds a history of handovers between BSs and uses this to determine which BS is the most likely neighbor target BS for a handover. Since the MOB_NBR-ADV messages gives the MS the list of neighbors and their parameters, knowing which BS is most likely the handover target improves the scanning operation.

Related works described in Section I-B either try to work around the required scanning by estimating how long it will take and then schedule the scanning operation efficiently or implement mechanisms such as new control messages or cross-layer handover techniques to improve upon the impact of scanning. We attempt to reduce the time required for the scanning operation. Our strategies require no additional network support and only limited memory and computational resources of the MS.

We tested our strategies by mapping real-world mobility data obtained via the APRS project [1] to simulate coverage area and performed simulations.

\section{B. Related Work}

There are two main complementary areas of research, that of efficiently scheduling the scanning operation and that of improving handover schemes. Neither attempt to reduce the number of frequencies checked during the scanning operation. Rouil and Golmie [9] recently introduced their Adaptive Channel Scanning (ACS) algorithm. ACS is primarily focused on when to perform scanning by estimating the time required for a MS to scan a list of neighboring BSs and then interleaving the scanning and data transmission intervals. Other work has been focused more specifically on improving handover performance. Choi et al. [5] have introduced a new management message to receive downlink data during the handover process and thus reduces the downlink packet delay. Kim et al. [8] proposed Last Packet Marking (LPM) that requires integrating the MAC layer (L2) handover and the network layer (L3) handover. LPM allows a MS to pre-notify a target BS for handover which the target BS can accept or reject.

An early work by Van de Berg [10] describes the storing information on the most probable used carrier frequencies in 
cellular networks on the MS . However, the term most probable is not defined and no mechanism is provided for determining the most probable frequencies.

The remainder of the paper is organized as follows. In Section II, we describe the IEEE 802.16 network entry procedure and handovers. We focus in particular on the scanning process. In Section III, we present the current IEEE 802.16e scanning operation and propose two new strategies. We provide a description of our simulation environment along with our simulation results in Section IV. Finally, we discuss ongoing work and conclude in Section V.

\section{Network Entry AND Handover Scanning IN IEEE $802.16 \mathrm{E}$}

The IEEE 802.16e/WiMAX standard defines a medium access control (MAC) layer as well as numerous underlying physical layer (PHY) specifications. The MAC protocol can be either time division duplex (TDD) or frequency division duplex (FDD) based. Communication between a mobile subscriber (MS) and the base station (BS) are established in a point-to-multipoint (PMP) architecture in a similar manner as traditional cellular networks.

The IEEE 802.16e standard defines a network entry procedure, depicted in Figure 1, for a MS wanting to establish a network connection via a BS. The MS must first scan to find a frequency in use by a BS. It does this by listening to each possible frequency until it hears the frame preamble. This takes a minimum of two frames [3], [9], at each channel. After finding the channel, it must synchronize with the BS by waiting for the Downlink Map (DL_MAP). The DL_MAP is a map of the timeslot locations in use for the frame. The maximum time between DL_MAPs can be as high as 11 seconds [3]. Once the MS has synchronized with the channel, it then must listen for the Downlink and Uplink Channel Descriptors (DCD and UCD) that are periodically sent using broadcast by the BS. Then the MS must wait for a contention slot (determined from the UCD) in order to perform Initial Ranging with the BS. Initial ranging is used to determine the transmit power requirements of the MS in order to reach the BS.

Since the network entry process has many steps, if we can improve upon the time it takes for one or more of these steps to complete we can provide an improved access time for initialization or recovery of service. In this paper, we focus on scanning.

\section{A. Scanning in IEEE 802.16e}

Scanning is an activity conducted by a MS. The goal of scanning is to acquire a downlink signal from a BS. Scanning is done by monitoring each possible frequency until a downlink signal is received. The exact number of frequencies depends on the regulatory provisioned bandwidth (varies from one country to another), physical specification (several) and bandwidth per channel (several options available per physical specification). Scanning is performed during the initial network entry procedure and continues periodically to

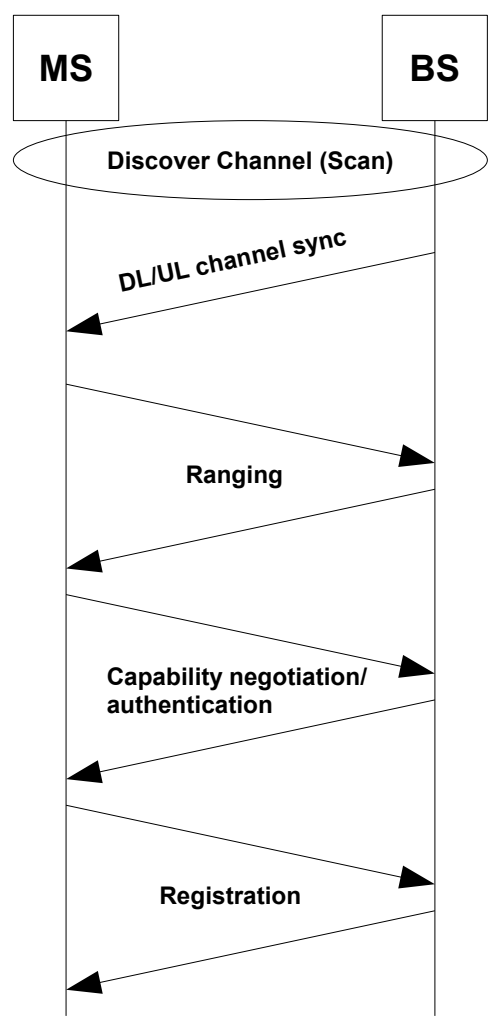

Fig. 1. IEEE 802.16e Network entry procedure.

aid the MS in the selection of a suitable target BS for a handover to maintain network connectivity while in motion.

The IEEE 802.16e specifications provide support for network assisted handovers where the BS currently serving the MS can obtain the information of neighboring BSs over the network. The serving BS periodically sends using broadcast this information as a MOB_NBR-ADV message to the MS.

Even though there is support for network assisted handovers, there are still scenarios where the ability of a MS to make its own decisions on scanning would improve performance. These include the following.

1) Initial Network Entry: here, the MS is not aware of its closest BS and must determine which frequency to use.

2) Handover from one BS to another BS where there is no network handover assistance provided - either it is not available, or the MS is moving between different service providers.

3) Handover between different network types (i.e. IEEE 802.11 to IEEE 802.16).

\section{B. IEEE 802.16e/WiMAX Handovers}

As a MS moves throughout the coverage area, maintaining connectivity is done via performing handovers between 
neighboring BSs. An example is shown in Figure 2, where a MS must choose one of six neighboring BSs, in this case neighbor six is chosen. Selection of the best handover target can be complex since the MS must scan for neighboring BSs to find a suitable target based on a number of criteria such as signal strength or error rates. Since a handover is an important function, a MS should perform the scanning and determine a target BS before beginning the handover. The IEEE 802.16e standard supports temporarily suspending the uplink and downlink communication between the MS and BS in order to allow the MS to perform scanning for neighboring BSs. While communication is suspended, the data streams must be buffered on either side. Any improvement on the time it takes for the MS to complete its scanning operation improves the performance of the communication, i.e. reduce delays.

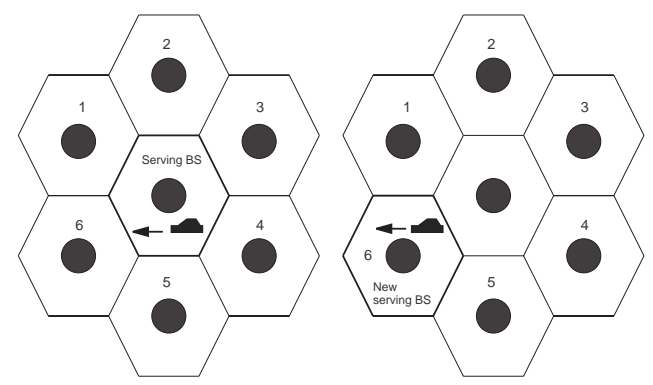

Fig. 2. Mobile station handover.

\section{SCANNING STRATEGIES}

In the following section, we describe the IEEE 802.16e default scanning strategy as well as two new strategies. The first new strategy, deals with reducing the number of frequencies checked during each scanning operation. The MS maintains information on the probabilities of the frequencies being used in order to make a choice. This strategy is further divided into the Most Recently Used and Most Frequently Used approaches. The second new strategy deals with the MS maintaining information on the history of performing handovers between BSs that were encountered under the assumption that patterns observed in handovers will be repeated.

\section{A. IEEE 802.16e Strategy}

The IEEE 802.16e standard [3] specifies that a MS must keep a nonvolatile storage where it stores the last set of operational parameters. When a MS wishes to acquire a downlink channel, it uses this stored information. Whenever a MS fails to obtain the downlink channel, it then continuously scans the possible channels of the downlink frequencies until a time it finds a downlink signal.

\section{B. Frequency Strategies}

When a MS is turned on for the first time, all frequencies are equally likely since the MS has no history. That is to say if there are $n$ frequencies, that the frequencies $f_{1}, f_{2}, \ldots, f_{n}$ have an initial probability distribution $p\left(f_{1}\right), p\left(f_{2}\right), \ldots, p\left(f_{n}\right)=$ $\frac{1}{n}$. This can be defined as a random variable $X$ assuming the values $f_{1}, f_{2}, \ldots f_{n}$ such that $\operatorname{Pr}\left[X=f_{i}\right]:=p\left(f_{i}\right)$. A MS only moves along a limited geometric planar region defined by the cells it traverses. This in turn gives rise to a random variable $X_{M S}$ induced by the random variable $X$ and having probability distribution $\operatorname{Pr}\left[X_{M S}=f_{i}\right]$. It is clear that this probability distribution will depend on the mobility characteristics (the path) of the MS as well as the probability distribution of the frequencies in the subregion traversed by the MS during its movements. Given this random variable, we are interested in optimizing how the MS selects its frequencies.

From the initial setup, since a MS has no previous history, it must simply start scanning frequencies in increasing order. As the MS performs a number of successful scanning operations, it can determine an order of frequencies from its observations. This history can be used to make scanning more efficient.

In the following sections we will discuss two new strategies that utilize the MSs history of scanning operations to determine the frequency ordering.

1) Most Recently Used Strategy (MRU): In this first strategy, the MS must keep a certain number of the possible frequencies stored in memory. This information is independent of BSs or network topology. Initially, all frequencies are equally likely and will be chosen in increasing order (lowest frequency to highest frequency). For each successful scanning operation, the frequency discovered, $f_{M R U_{1}}$, is given the highest priority, that is, it is moved to the front of the list of frequencies. As the MS builds up a history of scanning operations, it will have a frequency of occurrence, $F$, distribution as follows

$$
F\left(f_{M R U_{1}}\right)>F\left(f_{M R U_{2}}\right)>F\left(f_{M R U_{3}}\right)>\cdots>F\left(f_{M R U_{k}}\right)
$$

where $f_{M R U_{1}}$ is the most recently used frequency, $f_{M R U_{2}}$ is the second most recently used, and so on. The value of $k$ is the number of frequencies stored in memory. There is a training period of time required to establish this order. In future scanning operations, the MS scans frequencies in order from the most recently used (MRU) to the least recently used. If the MS does not find the frequency from the MRU list, then it must scan the remaining frequencies.

2) Most Frequently Used Strategy (MFU): In this strategy, a frequency of occurrence distribution over the frequencies is built based on the history of the number of times each frequency is used. The frequency that is used the most has the highest priority. As the MS builds up a history of scanning operations, the frequency of occurrence distribution will be as follows

$$
F\left(f_{M F U_{1}}\right) \geq F\left(f_{M F U_{2}}\right) \geq F\left(f_{M F U_{3}}\right) \geq \cdots \geq F\left(f_{M F U_{k}}\right)
$$

where $f_{M F U_{1}}$ is the most frequently used frequency, $f_{M F U_{2}}$ is the second most frequently used and so on. The value of $k$ is the number of frequencies stored in memory. The MS keeps an ordered list starting with the most frequently used frequency. In future scanning operations, the MS scans the frequencies from the most frequently used to the least frequently used frequencies. 


\section{Previous Handover Strategy}

In this section, we describe a new strategy that utilizes the history of the MS handovers along with the information made available from the currently serving $\mathrm{BS}$ in the MOB_NBRADV message to improve the choice of a handover target neighbour BS to begin scanning.

We assume the MS is provided with the list of all neighboring BSs and the frequency advertised for each neighbor in the MOB_NBR-ADV message from the serving BS. Even though this information is provided to the MS, the MS must "guess" at which of the neighboring BSs it should attempt to perform a handover. The MS must perform a scanning operation to obtain the operational parameters and determine which of the neighboring BSs are suitable for a handover. The IEEE 802.16e standard does not define this operation. It is left to the MS to decide on which neighbor to attempt to communicate. A MS with no other information than the MOB_NBR-ADV must simply attempt to scan for the neighbors in the order given.

That is to say that the neighbors for each $B S_{i}$, $n_{i 1}, n_{i 2}, \ldots, n_{i d}$ have an initial probability distribution $p\left(n_{i 1}\right), p\left(n_{i 2}\right), \ldots, p\left(n_{i d}\right)$. This can be defined as a random variable $Y$ assuming the values $n_{i 1}, n_{i 2}, \ldots, n_{i d}$ such that $\operatorname{Pr}\left[Y=n_{i j}\right]:=p\left(n_{i j}\right)$. We want to capture the probability that the MS currently served by $B S_{i}$ will perform a handover to $B S_{i}$ s neighbor $j$. Similarly to the frequency strategies, this probability distribution will depend on the mobility characteristics (the path) of the MS. Given this random variable, we are interested in optimizing how the MS selects its target handover BS. $p\left(H O_{i, j}\right)$ is the probability that a handover will occur from $B S_{i}$ to it's neighbor $B S_{j} . n$ is the number of BSs, and $d$ is the number of neighbors for $B S_{i}$.

The MS keeps for each $B S_{i}$ it has visited, a list of most probable handover target of the neighboring BSs. For example, a commuter driving along a highway is highly likely to repeatedly perform the same set of handovers along the highway, from $B S_{1}$ to $B S_{2}$ to $B S_{3}$ and so on. This pattern can be captured and stored in the MS. This can be done by maintaining a frequency of previous handovers that have occurred from each $B S_{i}$. The frequency of occurrence distribution, $F$, is as follows

$$
F\left(H O_{i, j_{1}}\right) \geq F\left(H O_{i, j_{2}}\right) \geq F\left(H O_{i, j_{3}}\right) \geq \ldots \geq F\left(H O_{i, j_{k}}\right)
$$

where we have non-increasing probabilities and $p\left(\mathrm{HO}_{i, j_{1}}\right)$ is the probability of a handover from $B S_{i}$ to one of its neighbors $B S_{j_{1}}$ which will be considered first when the MS performs scanning in preparation for a handover.

\section{Simulation}

In order to evaluate our strategies, we implemented a simulation to compare the IEEE 802.16e default scanning strategy with the $M R U$ and $M F U$ strategies. We then implemented a second simulation to test the previous handover strategy. We first describe important parameters concerning our simulation: 1) Network topology, 2) Metrics and measurements being used, 3) the Mobile Station Mobility Data used, and 4) Mobile
Station scan times. Later we will discuss the results of the simulation.

\section{A. Simulation Setup}

The simulations are setup as summarized in Table I. A ten kilometres by ten kilometres area is defined and covered by IEEE 802.16 base stations with a one kilometre range. The base stations are positioned based upon a cellular networking model [4] where each BS covers a hexagonal shaped region as shown in Figure 3. A total of $44 \mathrm{BS}$ are required to cover the entire area.

\begin{tabular}{|l|c|}
\hline \multicolumn{2}{|c|}{ Network Parameters } \\
\hline Coverage Area & $10 \mathrm{~km} \mathrm{x} 10 \mathrm{~km}$ \\
\hline No. Base Stations & 44 \\
\hline Cell Radius & $1 \mathrm{~km}$ \\
\hline Cell Cluster Size & 4 \\
\hline No. Frequencies & 20 \\
\hline No. Channels & 80 \\
\hline No. Channels per Cell & 20 \\
\hline No. Frequencies per cell & 5 \\
\hline
\end{tabular}

TABLE I

SIMULATION PARAMETERS

The WiMax simulation parameters are chosen based on 100 $\mathrm{MHz}$ of bandwidth available for both uplink and downlink channels. Every channel is given $1.25 \mathrm{MHz}$ of bandwidth for a total of 80 channels. A four cell cluster reuse clustering scheme, shown in Figure 4, is implemented on the underlying 44 BSs. This setup has 20 channels available for each cell for the 80 channels available in total. We use 20 different frequencies, assigning five to each BS, each of which has four channels for MS connections. Two MS scanning times are implemented in the simulation scenario.

In our model, the number of frequencies stored in memory is equal to the number of frequencies available in the coverage area.

Metrics and Measurement: The purpose of this work is to improve upon (reduce) the number of frequencies a MS is required to check while scanning for a downlink connection to a BS. We measure the proportion of time that a MS can find the downlink with a certain number of frequencies checked. For our model, this is between one and 20 frequencies checked per scan. Ideally, we would like to have it so that $100 \%$ of the time a MS can successfully scan for a neighboring BS with only checking a single frequency, but the actual performance is dependent on the individual MSs mobility. From the observations of our simulations, we determine the expected number of frequencies required to be checked for a certain proportion of scanning operations. For example, $x \%$ of the time, the MS can find a downlink channel with only scanning a single frequency. Results were obtained with a $95 \%$ level of confidence.

MS Mobility Data: We used real-world mobility data collected by the APRS project [1]. The data included the mobility traces of 50 different MSs. The actual real-world coordinates of the data for each MS were mapped to the simulation 


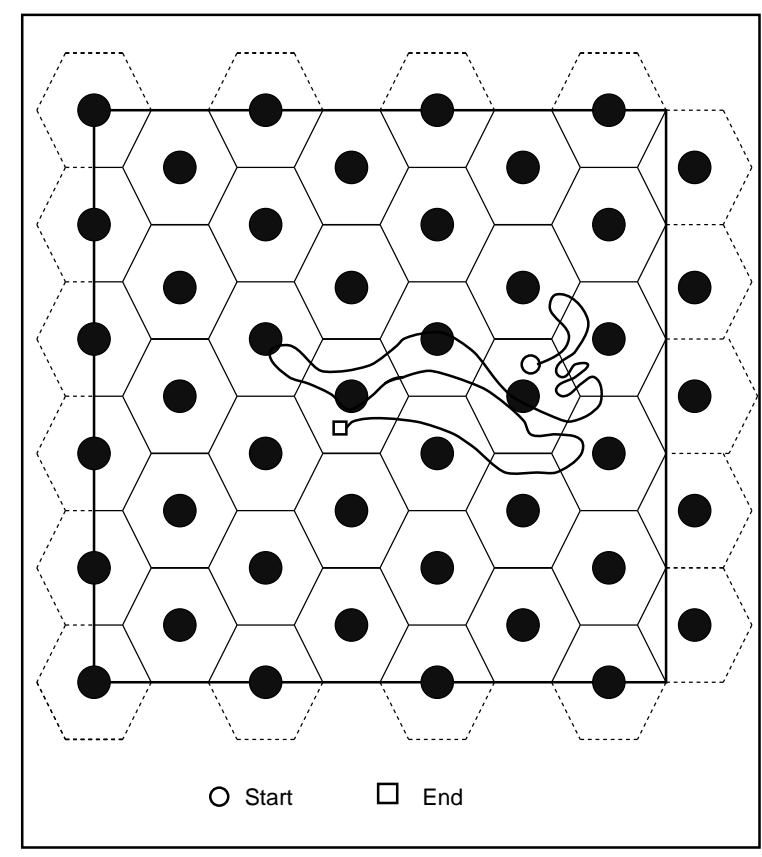

Fig. 3. Simulation area with sample path of MS.

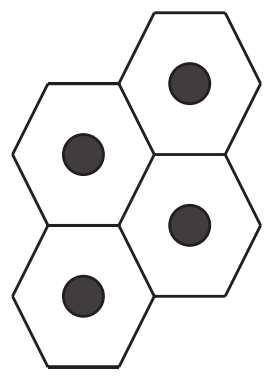

Fig. 4. Four cell cluster size for frequency reuse.

coverage area. As an example, a partial path of a sample MS is shown in Figure 3.

MS Scan Times: Here we describe two different scanning times of a MS. The first represents how scanning would be performed as a MS is in motion and performs handovers in order to maintain a network connection. The second represents the cases where a MS is just being turned on and must find a downlink from a BS to enter the network.

1) Scanning along the MS path: In this scenario, we chose sampled locations that were relatively close in time to the previous scan attempt. This would closely follow the path taken by the MS and would cover scenarios where the MS is trying to re-acquire a channel, or is performing a handoff between cells. We expected that the $M R U$ strategy would perform better in this scenario. Observe in Figure 3 that a MS may frequently move back and forth between the same series of two or more cells. It should be noted that the IEEE 802.16e default strategy is equivalent to the MRU strategy with a list of only one frequency.

2) Scanning based on random locations: In this scenario, we implement a series of randomly chosen sampled locations of each MS and perform a scan. This can be thought of to represent when a mobile station is turned on. It was expected that the $M F U$ strategy would achieve the most reduced number of scans with this scenario since a user can be expected to perform this kind of operation in certain places (i.e. home, office, etc.)

We performed simulations for the IEEE 802.16 default, $M R U$, and $M F U$ frequency strategies in order to compare their results.

Previous Handover Strategy: In this second simulation setup, the same area of coverage and mobility trace data were used. However, now the MS built a history of the previous BS handover pairs, and store for each BS, the list of neighboring BS in a $M F U$ order. That is, for each BS, the list of neighboring BSs will be maintained in the order of most often chosen as the handover target to the least often chosen as the handover target. When a MS receives a MOB_NBR-ADV message, it will choose the neighbor that is most frequently used first. From our simulation model, a MS will have at most six neighboring BSs from which they can choose for a handover. We performed simulations for the IEEE 802.16 default and the $M F U$ handover strategy in order to compare their results.

\section{B. Simulation Results}

The following sections show the simulation results of our frequency and handover strategies.

1) Frequency Strategies: Figure 5 gives the results of the scanning along the path scenario and shows the proportion of the time that a MS is required to scan a certain number of frequencies before finding the downlink signal from a BS. The results show that the $M R U$ strategy performs the best of the three strategies. Table II summarizes the results and can be read as follows: for our network parameters, the IEEE 802.16e default scanning strategey finds the downlink 51.9\% of the time by checking 10 frequencies. The MRU strategy finds the downlink $86.9 \%$ of the time with the same number of frequencies checked. In order to meet the MRU number, the default strategy requires checking 18 frequencies.

\begin{tabular}{|l|c|c|}
\hline \multicolumn{3}{|c|}{ Scanning along the path } \\
\hline & 5 Freq & 10 Freq \\
\hline Default & $39.9 \%$ & $51.9 \%$ \\
\hline MRU & $78.0 \%$ & $86.9 \%$ \\
\hline MFU & $53.2 \%$ & $79.5 \%$ \\
\hline
\end{tabular}

TABLE II

PROPORTION OF TIME SCANNING CAN BE COMPLETED WITH CHECKING 5, 10 FREQUENCIES

Similarly, Figure 6 shows the results for the random locations scenario. Here we observed that the MFU strategy performs best. The results are further summarized in Table III where we see that the MFU strategy successfully finds a BS frequency $79.5 \%$ of the time after checking 10 frequencies versus only $45.6 \%$ of the time for the IEEE 802.16 e default 


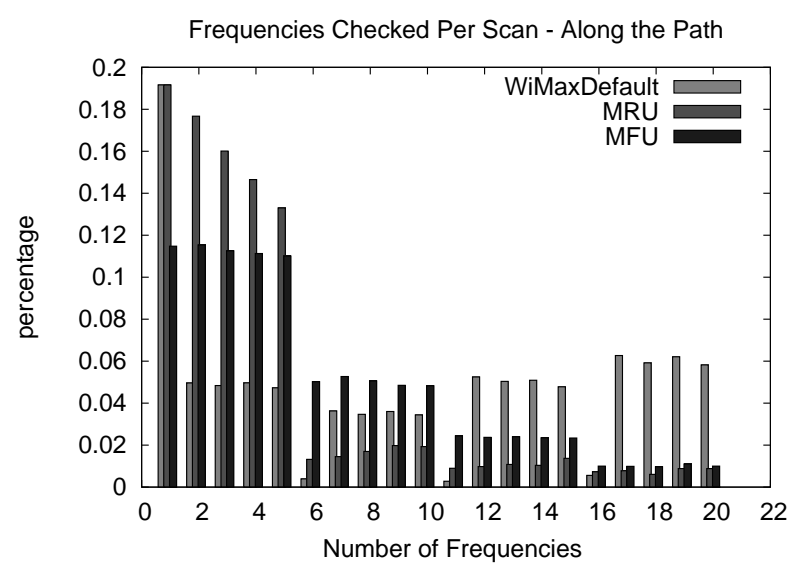

Fig. 5. Frequencies checked per scan - along MS path.

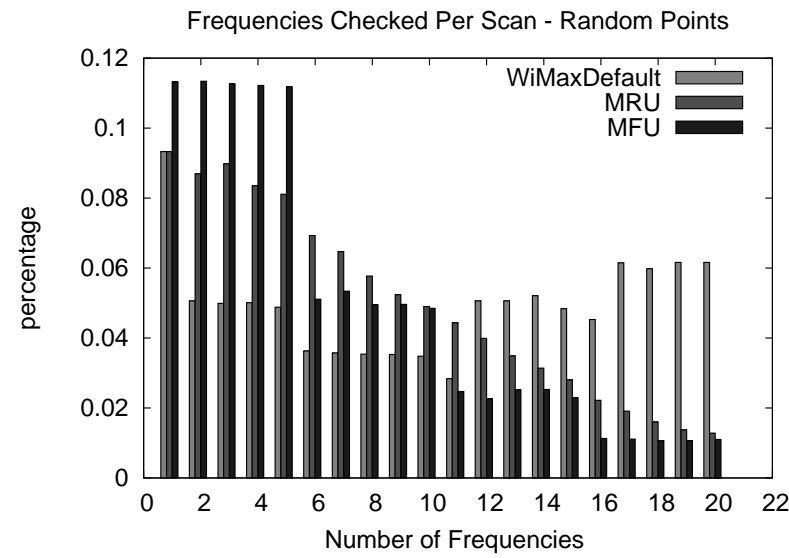

Fig. 6. Frequencies checked per scan - Random locations.

strategy. In order to meet the $79 \%$ of the MFU strategy, the default strategy is required to check 17 frequencies on average.

\begin{tabular}{|l|c|c|}
\hline \multicolumn{3}{|c|}{ Random points scenario } \\
\hline & 5 Freq & 10 Freq \\
\hline Default & $30.3 \%$ & $45.6 \%$ \\
\hline MRU & $40.0 \%$ & $70.7 \%$ \\
\hline MFU & $52.9 \%$ & $79.5 \%$ \\
\hline
\end{tabular}

TABLE III

PROPORTION OF TIME SCANNING CAN BE COMPLETED WITH CHECKING 5, 10 FREQUENCIES

2) Previous Handover Strategy: In this simulation scenario, the WiMAX default was to simply scan the neighbors in the order given by the MOB_NBR-ADV messages. By using information on the historical pattern of handovers from each BS, we obtain significant improvement in choice of neighbor BS frequency to scan. The results of the simulation are shown in Figure 7. We see that with the default strategy, slightly more than $20 \%$ of the time the MS picked the correct neighbor on the first attempt while with the MFU strategy this increases to over $70 \%$. While the WiMAX default was more evenly distributed, the MFU strategy successfully finds the correct handover target with only two attempts over $90 \%$ of the time.

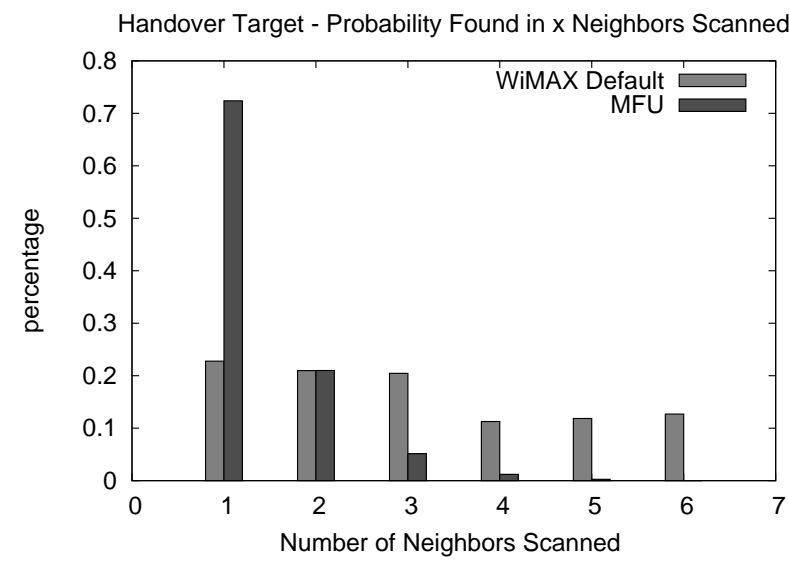

Fig. 7. BS neighbor scans per handover.

\section{CONCLUSion AND Future WorK}

In this paper we have examined the scanning procedure for an IEEE 802.16e MS. We have introduced two new strategies to aid in reducing the number of frequencies to check while scanning to find a downlink from a BS and provided some experimental results of a simulation based on real-world mobility traces. In the two scenarios we tested, along the path vs. random locations, we have shown that the $M R U$ (MFU respectively) performed best when compared against the default IEEE 802.16e scanning strategy. We introduced a second strategy to enhance the neighbor advertisement messages sent by the BS to assist the MS. In this strategy, the MS builds a history of previous handovers and uses this information to select which of the advertised neighboring BSs is most likely the correct one for a handover. Through our simulation, we have shown that this strategy improves performance significantly over that of the WiMAX default strategy.

Our work is complementary to the other research mentioned such as that done by Rouil and Gomlie [9]. Future work includes extended the model to include such things as (1) time of day (heading to the office, or home) (2) day of the week (weekday vs weekend) and (3) change of city (frequent business travel) parameters to further enhance performance. Finally, an additional refinement to the model to investigate how different loads on the WiMAX BSs may affect the scanning times.

\section{ACKNOWLEDGEMENTS}

Research supported in part by the Natural Sciences and Engineering Research Council of Canada (NSERC) and Mathematics of Information Technology and Complex Systems (MITACS). 


\section{REFERENCES}

[1] Filjar, R., Desic, S., Architecture of the Automatic Position Reporting System (APRS), 46th International Symposium Electronics in Marine (Elmar), pp. 331 - 335, 2004

[2] IEEE 802.16-2004, "IEEE Standard for Local and Metropolitan Area Networks - Part 16: Air Interface for Fixed Broadband Wireless Access Systems," 2004.

[3] IEEE 802.16e 2005, "IEEE Standard for Local and Metropolitan Area Networks - Part 16: Air Interface for Fixed and Mobile Broadband Wireless Access Systems - Amendment 2:Physical and medium access control layers for combined fixed and mobile operation in licensed bands annd corrigendum 1," February 2006

[4] D.P. Agrawal and Q-A. Zeng, Introduction to Wireless and Mobile Systems, 1st Edition, Thomson-Engineering, 2002.

[5] S. Choi, G. Hwang, T. Kwon, A. Lim, and D. Cho., "Fast handover scheme for Real-Time Downlink Services in IEEE 802.16e BWA System.”, In Vehicular Technology Conference, 2005. VTC 2005-Spring. pp. 2028-2032, January 2005.

[6] C. Eklund, R. B. Marks, K. L. Stanwood, and S. Wang, "IEEE Standard 802.16: A Technical Overview of the WirelessMAN ${ }^{\mathrm{TM}}$ Air Interface for Broadband Wireless Access," IEEE Communications Magazine, June 2002, pp. 98-107.

[7] H. Jang, J. Jee, Y-H. Han, S.D. Park, J.Cha, "Mobile IPV6 Fast Handovers over IEEE 802.16e Networks," MIPSHOP Working Group, Internet Draft, http://www.ietf.org/internet-drafts/draft-ietf-mipshop-fh80216e-01.txt

[8] K. Kim, C. Kim, T. Kim, "A Seamless Handover Mechanism for IEEE 802.16e Broadband Wireless Access," Proceedings of International Conference of Computational Science (ICCS) 2005.

[9] R. Rouil and N. Golmie, "Adaptive Channel Scanning for IEEE 802.16e," Proceedings of 25th Annual Military Communications Conference (MILCOM 2006), Washington, D.C., October 23-25, 2006.

[10] P. van de Berg, "Carrier Scanning in Cellular Networks," Patent number: WO/2000/078075, World Intellectual Property Organization, http://www.wipo.int, 2000 . 